\title{
The Cracked Mirror: Features of Narcissistic Personality Disorder in Children
}

\section{Karen Kernberg Bardenstein, PhD}

$\mathrm{T}$ questions regarding personality formation. According to the Diagnostic and Statistical Manual of Mental Disorders, fourth edition, ${ }^{1}$ personality is defined as "enduring patterns of perceiving, relating to and thinking about the environment and oneself ... when they are maladaptive and inflexible, they constitute Personality Disorders."1 Currently, such disorders are "generally recognized by adolescence or earlier." DSM-IV continues, however, by emphasizing corresponding diagnoses given to children and adolescents, such as Conduct Disorder, as an earlier equivalent to Antisocial Personality Disorder. Narcissistic Personality Disorder (NPD) has no such equivalent despite the acknowledgment that it develops earlier than 18 years. The ambivalence and ambiguity regarding personality disorder in children are further highlighted by the suggestion that one can apply the diagnosis of personality

Karen Kernberg Bardenstein, $P h D$, is with Case Western Reserve University, Cleveland, $\mathrm{OH}$.

Address correspondence to: Karen Kernberg Bardenstein, PhD, 12429 Cedar Road, Suite 18, Cleveland Heights, $\mathrm{OH} 44106$; e-mail kkbard@earthlink.net

Dr. Bardenstein has disclosed no relevant financial relationships.

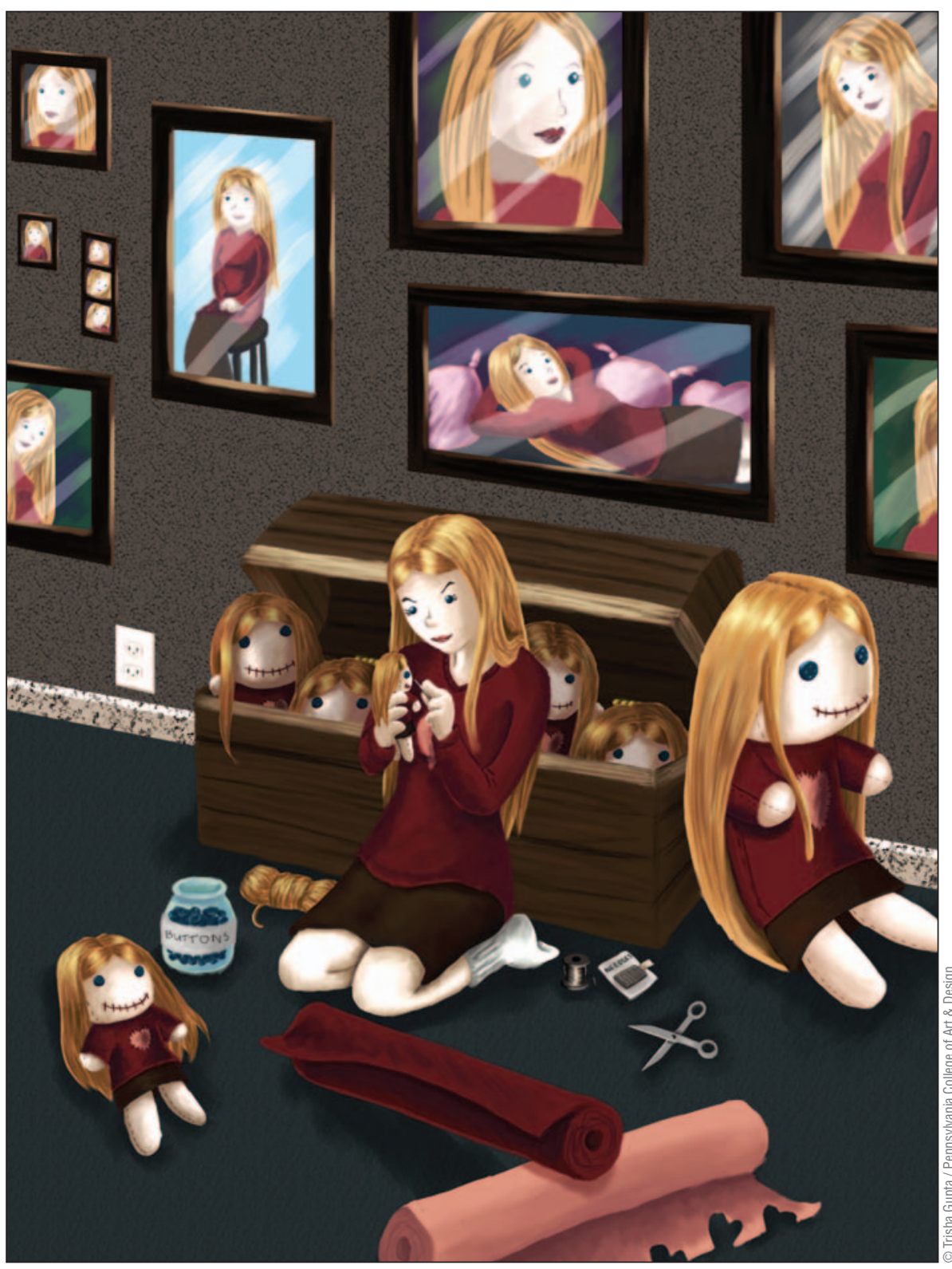

PsychiatricAnnalsOnline.com | 147 
disorder to children if the traits are stable for at least 1 year and are not limited to a particular developmental stage.

\section{NORMAL AND PATHOLOGICAL NARCISSISM IN CHILDREN}

P. Kernberg ${ }^{2}$ contrasts normal narcissism from pathological NPD traits in children in the following ways. The normal child's need for admiration is satisfied by the age-appropriate attention that he receives. He is able to express gratitude for and can reciprocate what others give him. Such children genuinely value and love the significant people in their lives. Normal children do have fantasies of being powerful, famous, and highly successful. They imagine themselves as president, a famous actress or athlete, or even a hero with superpowers. The distinguishing feature of such wishes from pathological narcissism, however, is the awareness that the wish is an aspiration and that others have the ability to be special as well. The narcissistic child is convinced that he is already endowed with unique and special abilities and becomes envious if anyone else becomes successful. One 4-year-old adopted girl was enraged that newborn twin baby sisters arrived, and she commanded all the attention. When a fellow classmate was praised for a drawing, this girl grabbed the drawing and ripped it up. Narcissistic children are in need of constant admiration and assurance that they are uniquely special.

Normal children's needs are realistic and can be fulfilled. They show genuine attachment to friends and family and trust significant adults in their lives. Their capacity to maintain good self-esteem, empathy, and consideration of others sets them apart from their narcissistic counterparts. Self-esteem refers to a satisfaction with one's self and how one lives one's life. ${ }^{3}$ It involves an evaluation of how one sees and feels about the self. Well-adjusted children are able to accept themselves, flaws and all, and can be resilient in the face of disappointment or failure. They can lose at board games or suffer the loss of a championship without becoming devastated or enraged. They do not take such temporary setbacks as an assault to their self worth, in contrast to the narcissistic child. Researchers have

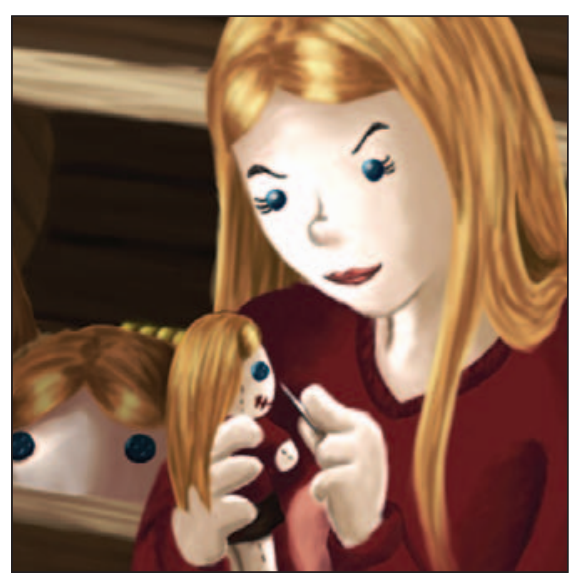

\section{Normal children do have fantasies of being powerful, famous, and highly successful.}

documented that narcissism and high self-esteem are independent constructs in child development and that narcissism (in contrast with high self-esteem) in children correlates with other pathological behavior. ${ }^{4-6}$

The literature supporting the presence of narcissistic pathology in youth continues to expand. Bleiberg ${ }^{7-9}$ articulated narcissistic pathology in children, based on his clinical experience. He integrated theory and research about constitutional factors, attachment disorders, and trauma. He contends that narcissistic children have a fundamental deficit in their capacity for "reflective functioning." This capacity refers to the ability to interpret accurately and respond adaptively to the world. Impairment interferes with the capacity to intuit other's intentions and empathetically grasp the feelings, thoughts, and motivations that underlie others' behavior as well as one's own.
Paulina Kernberg ${ }^{2,10}$ pioneered the application of NPD criteria and dynamics as developed by Otto Kernberg ${ }^{11}$ to children and added additional descriptive characteristics. The narcissistic child has a grandiose sense of self as evidenced by difficulty tolerating anything in which he is not immediately successful. Failure to maintain efforts in academic work or activities involving learning new skills is a common feature. The inflated sense of self impairs the development of a normal conscience (or superego) since any acknowledgment of one's flaws or failure to meet expectations is unbearable. Such children cannot experience guilt nor concern about the affect of their behavior on others. A 10-year-old patient remarked when asked about his reaction causing a concussion in his fellow teammate, "Well, he just happened to be in the wrong place at the wrong time and got hit in the head when I kicked the ball." The narcissistic child justifies personal deficits, irresponsible behavior or defeats through blaming others, evasively responding or outright lying. P. Kernberg noted that her narcissistic child patients developed both antisocial traits and paranoid anxiety due to this impairment in ego functioning. . $^{210}$

Kernberg observed that the sense of entitlement, stemming from their sense of superiority, leads to exploitation of others. An 11-year-old narcissistic patient asserted that she wanted to "grow up and be rich and have slaves." In contrast to the normal child, the narcissistic child feels entitled to what is received and the need to be grateful or reciprocate is compromised. The constant need to fuel the brittle sense of self and protect it from external assaults results in extreme distrust of others and rage when challenged or criticized. These children often crave material goods and quickly tire of and devalue what they have already been given. One 9-year old girl demanded that she get presents and attention on her mother's birthday. Like their adult counterparts, these children exhibit intense envy of 
others, devaluation, lack of empathy, and the inability to express gratitude or concern for others. A 13-year-old patient remarked that one apologizes in order to maintain one's image and "not let them think that you enjoy hurting people." He also defined the word "obey," as "listening to the whims of others." Another boy of 14 years attempted to write a letter of apology for misbehavior to his mother but quickly shifted to demanding that she lavishly redecorate his room.

Kernberg summarizes the childhood context in which narcissistic pathology becomes evident. Peer relationships are compromised by the lack of empathy, the need to be exploitative, devaluing, and manipulative. The quality of the friendships is superficial. One young patient boasted that he has " 1,000 friends" but could not name anyone who knew him well or whom he trusted. These children often become bossy and coercive with friends. Their arrogance interferes with the ability to take turns, comply with their friends' wishes, or follow agreedupon game rules.

Academic performance also suffers because narcissistic children do not enjoy their learning experiences. Achievement serves the purpose of eliciting admiration rather than acquiring knowledge for its own intrinsic value. If admiration wanes, the child becomes easily bored. Despite innate intelligence in some narcissistic children, they lose motivation to apply themselves in new subjects and skills and get poor grades because of to lack of effort. Kernberg also noted their inability to maintain eye contact as a defense against potential critical scrutiny and "tuning out" of the unwelcome demands or expectations of others. They are so haughty that no one can tell them what to do, and their capacity to learn from others' feedback is curtailed.

Additional pathology is evident in the narcissistic child's play. The child initially professes boredom, dissatisfaction with the toys, or devaluation of games. Kern- berg interprets such behaviors as defenses against sadistic scenarios of primitive aggression, potential failure to perform well with games, and exposure of any deficits to the therapist. She notes that as treatment progresses, sadistic fantasies of destroying rivals, being invulnerable and controlling and devaluing others (including the therapist) begin to appear.

\section{ORIGINS OF NARCISSISTIC PATHOLOGY IN CHILDREN}

P. Kernberg ${ }^{10}$ described the possible etiology of pathological narcissism in children. From her clinical experience, she identified certain circumstances that increase the risk of narcissistic pathology: the child of narcissistic parents, the adopted child, the child of successful parents (particularly if the child lacks similar ability), the overindulged or wealthy child, and the child of divorce. Narcissistic parents may over-idealize their children and insulate them from disappointment or criticism. The children can easily develop the idea that they are, in fact, superior to others and above criticism and failure. One couple, convinced that their 5-year-old was a genius, removed him from consecutive schools they deemed incompetent and mediocre because he was not receiving teachers' praise. They dismissed the school's concerns about the child's aggressive behavior as irrelevant and justified his poor adjustment as boredom. When they received a report that the child tested as having average intelligence, they complained to the head of the psychiatric hospital about the incompetence of the evaluator.

Children who are adopted can be susceptible because they have to address the initial rejection of why their biological parents did not keep them. Adoptive parents may compensate for this injury by emphasizing how they are more special than biological children because they were chosen, especially if biological siblings are part of the family. One of Kern- berg's child patients announced that if he had not been adopted by his current parents, another set of parents would have adopted him because he knew that there were so many parents in line that would have been eager to have him. Adoptive parents may also overcompensate for their sense of damage at not being able to produce a biological child by overindulging the adoptive child.

Children of the wealthy, or who have been overindulged, may be raised in an environment where entitlement and control of others is accepted and reinforced. Wealthy parents may also be invested in protecting the child from disappointment and accommodate to his wishes. These children may expect to have the best and internalize the devaluation of more common lifestyles. The author's child reported that a fellow camper at an overnight summer camp complained that the bathrooms were not made with marble floors and walls and that he would not return to camp again. Deferential treatment of the child because of his connections can further fuel grandiosity.

Children of successful parents may have the expectation that they should naturally be as talented without effort because they born from brilliance. Meeting such expectations is particularly difficult if the child has less ability or has any deficits that affect success. Narcissistic pathology serves as a compensation for a sense of inadequacy that is too painful to acknowledge. The son of a famous basketball player failed to be accepted in his school basketball team and responded with severe tantrums and devaluation of the coach.

Children of divorce are particularly susceptible if the parents convey that they are prized possessions that are vied for. Each parent in an attempt to carry favor with the child may be at risk for not providing critical feedback and refusing to indulge the child with material goods or excessive privileges. In turn, some children of divorce develop a sense of self- 
importance, entitlement, and devaluation of the parent who does not offer immediate gratification or flexible limits.

Parent-child interactions continue to be studied with respect to their contribution to narcissistic pathology and are critical aspects of intervention with narcissistic children. ${ }^{2,12}$

\section{ASSESSMENT OF NARCISSISTIC PATHOLOGY IN CHILDREN AND ADOLESCENTS}

Egan and Kernberg, ${ }^{13}$ Ferreira, ${ }^{14}$ Rinsley, ${ }^{15}$ Cohen, ${ }^{16}$ Beren, ${ }^{17}$ Bernstein, ${ }^{18}$ and Imbesi ${ }^{19}$ wrote about narcissistic traits in children from the psychoanalytic perspective as well. They proposed theoretical explanations for the distinctive characteristics of the disorder including grandiosity, negative and aggressive transference, attachment difficulties, boundary disturbances between self, and other and primitive defenses such as splitting and devaluation. These publications are primarily based on clinical observations of child patients in treatment. Bleiberg ${ }^{9}$ integrated his previous work with additional clinical material and treatment considerations but did not address psychological testing of such children.

Guile $^{20}$ assessed the three different systems for diagnosing narcissism in children based on DSM diagnostic criteria (P. Kernberg's for children, ${ }^{2}$ Bleiberg's for adolescents, ${ }^{8}$ and DSMIV criteria for pre-adolescents ${ }^{1}$ ) and reported a high concordance rate. $\mathrm{He}$ concluded that NPD could be identified among pre-adolescents. With the increasing ability to consistently identify narcissistic features, the research on narcissistic personality in children, especially using psychological instruments and structured interviews, has expanded as a result. Kernberg, Weiner and Bardenstein ${ }^{2}$ reported psychological test findings that were characteristic of children with personality disorder in general, as well as narcissistic personality disorder.
Some studies have utilized structured interviews to systematically identify narcissistic features in children. Eppright et $\mathrm{al}^{21}$ interviewed incarcerated adolescents with the Diagnostic Interview for Children and Adolescents-Revised and the Structured Clinical Interview for Mental Disorders-III-Revised, for Personality Disorders, to establish diagnoses. They observed that after antisocial personality disorder, narcissistic personality disorder was one of the most frequent diagnoses. Myers et $\mathrm{al}^{22}$ similarly conducted structured diagnostic interviews and utilized the Revised Psychopathy Checklist (PCL-R) to evaluate comorbid personality disorders in psychiatrically hospitalized adolescents and reported significant relationships between psychopathy scores and narcissistic personality disorder.

Recent reports are documenting the validity and stability of personality disorder in children and adolescents using behavioral checklists and interviews. 17,20,23,24 $^{2}$ The Narcissistic Personality Inventory developed by Raskin and $\mathrm{Hall}^{25}$ has been applied to children and adolescents. ${ }^{12,26}$ $\mathrm{Ang}^{27}$ has recently reported on the Narcissistic Personality Questionnaire for Children-Revised (NPQC-R), which has adequate reliability and validity to serve as a measure of superiority and exploitation. Thomaes ${ }^{28}$ introduced the Childhood Narcissism Scale, which also validly and reliably assesses grandiose sense of self, inflated sense of superiority and entitlement, and exploitative interpersonal attitudes. Guile ${ }^{24}$ reported adequate reliability for the Diagnostic Interview for Narcissism Adapted for Pre-Adolescents: Parent Version (P-DIN), based on Gunderson's Diagnostic Interview. ${ }^{29}$ These instruments are providing further understanding about how narcissism interacts with aggression, delinquency, shame, and interpersonal adjustment in young populations. ${ }^{5,6,26,30-33}$ Crawford $^{33}$ reported high stability of pathological narcissistic behaviors in children from early adolescence into adulthood.
Psychological testing has only been recently applied to the study of narcissism in children. Abrams ${ }^{34}$ reports a single case study using the Thematic Apperception Test (TAT) and the Children's Apperception Test (CAT) to assess an 8year-old boy and describes his dynamic issues. The TAT and the CAT, however, lack the rigorous empirical validity and reliability required to systematically study personality disorder, despite their rich clinical utility.

Although research examining character or personality disorder in adults has become common in the Rorschach literature, a notable absence exists regarding characteristics of children with personality disorders. The Rorschach's ability to methodically and validly assess narcissistic personality disorder has been established by Hilsenroth and colleagues, ${ }^{35}$ utilizing independently diagnosed patients based on DSM-IV criteria $^{1}$ for Clusters A, B, and C personality disorders. (Previous publications reporting the Rorschach's ability to differentiate NPD from other personality disorders were not based on DSM-IV criteria.) They demonstrated that the Rorschach variables of reflection (one image is mirrored by another), pairs (two related or identical items are seen), personalization (the response is justified not be aspects of the inkblot but by personal experience), idealization (aggrandizing attributes of the percept), and the Egocentricity Index (a ratio of reflection and pair responses to total number of responses) effectively differentiated the NPD group from a non-clinical sample and from Cluster A, Cluster C, and other Cluster B personality disorders. They noted two variables that were robustly significant across statistical analyses: reflection responses and idealization. The Egocentricity Index and pair responses differentiated NPD from some groups but not consistently across all groups. The authors found significant correlations between Rorschach criteria and DSM-IV criteria for NPD. 


\section{LEXAPRO ${ }^{\circledast}$ (escitalopram oxalate) TABLETS/ORAL SOLUTION}

(5\% and 4\%); Fatigue (5\% and 2\%). Psychiatric Disorders: Insomnia (9\% and 4\%); Somnolence (6\% and 2\%); Appetite Decreased (3\% and 1\%); Libido Decreased (3\% and 1\%). Respiratory System Disorders: Rhinitis (5\% and 4\%); Sinusitis (3\% and 2\%). Urogenital: Ejaculation Disorderr.2 $(9 \%$ and $<1 \%$ ) Impotence $23 \%$ and < $1 \%$ ); Anorgasmias $32 \%$ and < $1 \%$ ). ${ }^{*}$ Events reported by at least $2 \%$ of patients treated with Lexapro are reported, except for the following

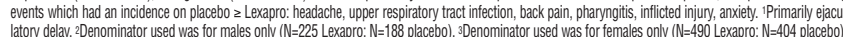
latory delay. 2Denominator used was for males only (N=225 Lexapro; N=188 placebo). SDenominator used was for females only (N=490 Lexapro; $N=404$ placebo).
Generalized Anxiety Disorder Table 3 enumerates the incidence, rounded to the nearest percent of freatment-emergent adverse events that occurred among 429

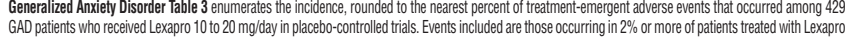
GAD patients who received Lexapro 10 to $20 \mathrm{mg}$ dad ay in placebo-controlled trials. Events included are those occurring in $2 \%$ or more of patients treated with Lexapo

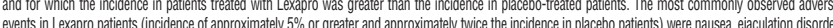
(primarily ejaculatory delay), insomnia, fatigue, decreased libido, and anorgasmia (see TABLE 3). TABIE 3: Treatment-Emergent Adverse Events: Incidence in Placebo-Controlled Clinical Trials for Generalized Anxiety Disorder * (Percentage of Patients Reporting Event) Body System/Adverse Event ILexapro (N=429) and Placebo (N=427): Autonomic Nervous System Disorders: Dry Mouth (9\% and 5\%); Sweating Increased (4\% and 1\%). Central \& Peripheral Nervous System Disorders: Headache (24\% and 17\%); Paresthesia (2\% and 1\%). Gastrointestinal Disorders: Naussea (18\% and 8\%); Diarrhea (8\% and 6\%); Constipation (5\% and 4\%); Indigestion (3\% and 2\%); Vomiting (3\% and 1\%); ; Addominal Pain (2\% and 1\%); Flatulence (2\% and 1\%); Toothache (2\% and $0 \%$ ). General

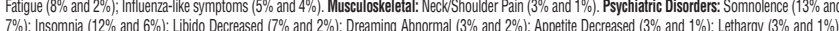
7\%); Insomnia (12\% and 6\%); Libido Decreased (7\% and 2\%); Dreaming Abnormal (3\% and 2\%); Appetite Decreased (3\% and 1\%); Lethargy (3\% and 1\%

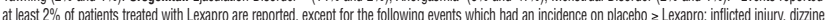

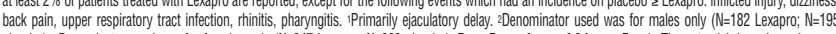
placebo). 3Denominator used was for females only ( $\mathrm{N}=247$ Lexapro; $\mathrm{N}=232$ placebo). Dose Dependency of Adverse Events The potential dose dependency of common adverse events (defined as an incidence rate of $>5 \%$ in either the $10 \mathrm{mg}$ or $20 \mathrm{mg}$ Lexapro groups) was examined on the basis of the combined incidence of adverse events in two fixed-dose trials. The overall incidence rates of adverse events in $10 \mathrm{mg}$ Lexapro-treated patients $(66 \%)$ was similar to that the placebo-treated patients (61\%), whilie the incidence rate in 20 mg/day Lexapro-treated patients was greater ( $86 \%$ ). Table 4 shows common adverse events that occurred in the 20 mglday Lexapro group with an incidence that was approximately twice that of the $10 \mathrm{~m} /$ dday Lexapro group and approximatele twice th

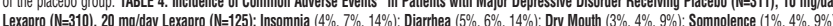

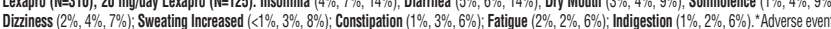
with an incidence rate of at least $5 \%$ in either of the Lexapro groups and with an incidence rate in the 20 mglday Lexapro group that was approximately twic that of the $10 \mathrm{mg}$ day Lexapro group and the placebo group. Male and Female Sexual Dystunction with SSRls Atthough changes in sexval desire, sext performance, and sexual satisfaction often occur as manifiestations of a psychiatric disorder, they may also be a consequence of pharmacologic treatment. particular, some evidence suggestst that SSRIs can cause such untoward sexual experiences. Reliable estimates of the incidence and severity of untoward exper ences involving sexual desire, performance, and satisfaction are dificicult to obtain, however, in part because patients and physicians may be reluctant to discus incidence. Table 5 shows the incidence rates of sexval side effects in patients with maior depressive disorder and GAD in placebo-controlled trials. TABLE 5 . Incidence of Sexual Side Effects in Placebo-Controlled Clinical Trials [In Males Only: Adverse Event: Lexapro ( $\mathrm{N}=407$ ) and Placebo (N=3833]) Eacculation Disorder (primarily ejaculatory delay) (12\% and 1\%); : Libido Decreased (6\% and 2\%); ; Impotence (2\% and <1\%). [I Females Only: Lexapro (N=737) and Placebo $(\mathrm{N}=636)$ ): Libido Decreased $(3 \%$ and $1 \%$, Anoryasmila $(3 \%$ and $<1 \%$ ) There are no adequately designed studes examining sexual dysfunction with escitalopran

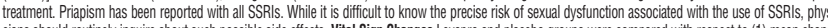
cians should routinely inquire about such possible side effects. Vital Sign Changes Lexapro and placebo groups were compared with ressect to (1) mean chang significant changes from haseline in these variables These analyses did not revel any clinically important changes in vital signs associated with Lexapro traer ment In addition a comparison of supine and standing vital sign measures in subjects receiving Lexapro indicated that Lexapro treatment is not associated wi orthostatic changes. Weight Changes Patients treated with Lexapro in controlled trials did not difier from placebo-treated patients with regard to clinically important change in body weight. Laboratory Changes Lexapro and phacebo groups were compared with respect to (1) mean change from baseline in various serr. chemistry, hematology, and urinalysis variables, and (2) the incidence of patients meeting criteria for potentially clinically significant changes srom baseline these variables. These analyses revealed no clinically important changes in laboratotory test parameters associated with Lexapro treatment. ECG Changes

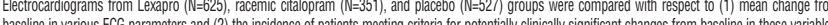
Daseline in various ECG parameters and (2) the incidence of patients meeting criteria tor potentially clinically significant changes from baseline in these variables, placebo and (2) an increase in QTc interval of $3.9 \mathrm{msec}$ for Lexapro and $3.7 \mathrm{msec}$ for racemic citalopram, compared to $0.5 \mathrm{msec}$ for placebo. Neither Lexapro nor
racemic citalopram were associated with the development of clinically significant ECG abnormalities. Other Events Observed During the Premarketing Evaluation of Lexapro Following is a list of WHO terms that reflect treatment-emergent adverse events, as defined in the introduction to the ADVERSE REACTIONS section reported by the 1428 patients treated with Lexapro for periods of up to one year in double-blind or open-label clinical trials during its premarketing evaluation Ail reported eventis are included except those aready isted in Tables $2 \& 3$, those occurring in only one patient, event terms that are so generala as to be uninifor

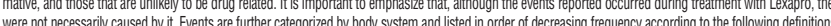
frequent adverse events are those occuring on one or more occesions in at least $1 / 100$ patients intrecuent adverse events are those occurring in less than $1 / 100$ vein. Central and Peripheral Nervous System Disorders - Frequent: lighth-headed feeling, migraine. Infrequent: tremor, vertigo, restless legs, shaking, twitching dysequilibrium, tics, carpal tunnel syndrome, muscle contractions involuntary, suggishness, coordination abnormal, faintness, hyperretexia, muscular ton increased. Gastriointestina Disordetrs - Frequent. heartburn, abdominal cramp, gastrioentertits. Infrequent: gastroesophageal reflux, bloating, abdominal discon tott, dyspepssia, increased stool frequency, belching, aastritis, hemorrhoids, gagging, polyposis gastric, swallowing dificicut. General - Frequent: allergy, pain in limb

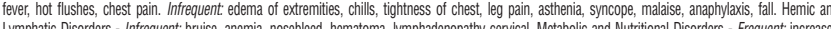

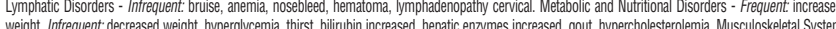

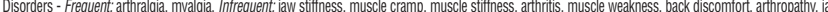
pain, joint stiffness. Psychiatric Disorders - Frequent: appetite increased, lethargy, irritability, concentration impaired. Infrequents: itteriness, panic reaction, agitation, apathy, forgettulunss, depression aggravated, nervousness, restlessness aggravated, suicide attempt, amnesia, anxiety attack, bruxism, carbohydra

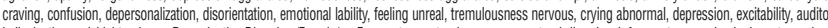

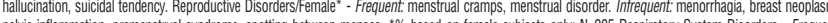

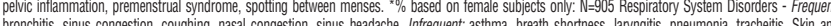

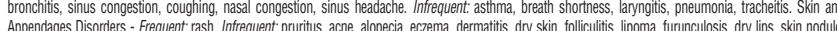

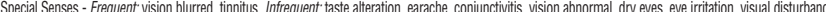
eye infection, pupils dilated, metallic taste. Urinary System Disorders - Frequent urinary frequency, urinary tract tinfection. Infrequent urinary urgency, kidney stone dysuria, blood in urine. Events Reported Subsequent to the Marketing of Escitalopram - Atthough no causal relationship to escitalopram treatment has be found, the tollowing adverse vents have been reponted to have occurred in pateints and to be temporally associaled with escitalopram treatment during pos marketing spontaneous and clinical trial experenence and were not observed during the premarketing evaluation of escitalopram: Blood and Lymphatic Syster Disorders.

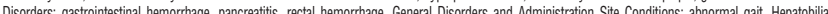
Disorders: fulminant henatitis, henatic failure, hepatic necrosis, henatitis Immune System Disorders: allergic reaction Investigations: electrocardiooram 0 prolongation, INR increased, prothrombin decreased. Metabolism and Nutrition Disorders: hypoglycemia, hypokalemia. Musculoskeletal and Connective Tiss Disorders: hrabdomyolysis. Nervous System Disorders: akathisia, choreoathetosis, dysarthria, dyskinesia, dystonia, extrapyramidal disorders, grand mal seizures (or convulsions), hypoaesthesia, myocionus, neuroleptic malignant syndrome, nystagmus, seizures, serotonin syndrome, tardive dyskinesia. Pregnane

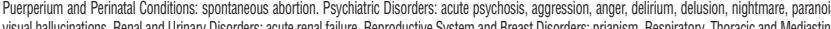

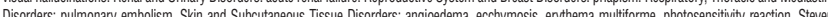

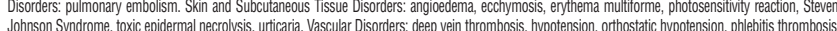
Forest Pharmaceuticals, Inc. Subsidiary of Forest Laboratories, Inc. St. Louis, MO 63045 USA Licensed from H. Lundbeck AS Rev. 04/08 @2008 Forest Laboratories, Inc.
The Rorschach is clearly a compelling instrument with which to assess "patterns of perceiving, relating to, and thinking about the environment" but has yet to be actively utilized to address the issue of whether children exhibit personality disorder and if such disorders truly persist over time. Although numerous Rorschach indices and features have been proposed as indicative or characteristic of adult NPD, such as the reflection response, ${ }^{36}$ most examine the content of the response (as opposed to the coding of the response). Kwawer ${ }^{37}$ notes that "narcissistic mirroring" in responses involving reflection, twin imagery or shadows is associated with narcissistic traits. Lerner and Lerner ${ }^{38}$ and Cooper, Perry, and Arnow ${ }^{39}$ infer narcissistic defenses, devaluation, and idealization in the content of the responses as well. Berg ${ }^{40}$ examines grandiosity in the content. Wagner and Hoover ${ }^{41}$ report exhibitionism as indicative of the narcissistic protocol. The exploratory nature of Rorschach assessment of NPD in children required a pilot study to examine what structural features would be generated by children and adolescents who had narcissistic clinical presentations. The current study was developed to test whether previously identified features in NPD adults would be similarly present and whether other features would distinctly characterize these younger patients.

The particular questions addressed in the study include whether any structural features would emerge consistent with theoretical explanations, whether chronicity of the features would be evident (which would support the notion of an enduring constellation of traits), and whether the children and adolescents of the sample would share a distinct constellation of traits that could be contrasted with other child patient populations.

\section{RORSCHACH FEATURES OF NPD CHILDREN}

The initial study included the Rorschachs of 36 children, ${ }^{42}$ ranging in age from 5 to 17 years, with a mean age of 11.8 years. The sample was divided approximately equally among female and male patients, with the majority coming from middle to upper class backgrounds. The patient group was mostly white, $(89 \%)$ with two black and three Asian patients. All the children were evaluated in an outpatient setting over a period of 5 years and presented behaviorally as NPDs as defined by DSM-IV criteria, including a pervasive pattern of grandiosity, lack of empathy, and hypersensitivity to criticism.

The childhood equivalents included: temper tantrums when criticized or made demands of; antisocial behavior such as lying, stealing, or physical aggressiveness with others; devaluation of others; envy of others' status, material wealth, or skills; grandiose self perceptions not founded in reality; suspiciousness of others' motives; denial or lack of 


\section{SIDEBAR}

\section{DSM-IV Criteria for Narcissistic Personality Disorder}

1. Grandiose sense of self-importance.

2. Preoccupation with fantasies of unlimited success, power, brilliance, beauty, or ideal love.

3. Belief that one is special, unique, and can only be understood by or should associate with other special or high-status people.

4. Requiring excessive admiration.

5. Sense of entitlement, unreasonable expectations of especially favorable treatment, or automatic compliance with expectations.

6. Interpersonal exploitation.

7. Lack of empathy.

8. Being envious of others and believing others are envious in turn.

9. Arrogant, haughty behaviors and attitudes.

responsibility for one's actions; and a lack of remorse and concern for others. These behaviors are included in the Hare Psychopathy scale ${ }^{43}$ for the differential diagnosis of NPDs with antisocial features. One child had retorted to his art teacher after being reprimanded, "I'd rather clean the toilets in the bathroom than do your project." Another child had threatened to sue his school for "harassment" because he lost credit when he failed to turn in an assignment. A first grader told his teacher that she was "obtuse," and he would prefer to count bricks in the hall than to stay in class with her. A second grader refused to do her worksheets and explained to her teacher that she did not have to do such boring work in summer camp or Sunday school, so she did not have to do it in regular school.

These children were characterized by previous resistance to treatment interventions, oppositional and defiant behavior, lack of motivation to pursue activities or school when success was not immediately forthcoming, expectations that others are there to gratify needs without a need for reciprocity or gratitude, a constant need to be the center of attention, and a lack of investment in friends or family, resulting in superficial relationships. They externalized blame for their behavior to avoid punishment or justify poor work. All of the patients were indifferent to the dis- tress caused by their maladaptive behavior. The DSM-IV criteria are summarized in the Sidebar.

The Rorschach protocols were scored by two raters using the Exner Comprehensive System ${ }^{44}$ with acceptable reliability (91\%). The second rater was blinded to the diagnoses of the patients. The Rorschachs were then scored for structural features and reviewed for those that occurred in more than half the protocols and were also significant $(>1$ standard deviation) from the non-patient normative data published by Exner ${ }^{44}$ for the comparable age. Although Exner generally discourages such a practice, he allows for reports that are highly discrepant from the expected range. The results were divided into smaller groups based on age to also reveal possible developmental indications that would be otherwise lost by combining latency age children with older adolescents.

The Rorschach findings were clustered along basic aspects of personality functioning including: 1) constellations of clinically related traits; 2 ) the experience and expression of emotions; 3 ) cognitive functions; 4) interpersonal perceptions, and 5) self-perception. The explanations of the terms will appear in the discussion of the results.

These young narcissistic patients tend to be positive on several of the Exner constellations. They exhibited a capac- ity to distort reality, engage in peculiar or thought-disordered ideation, and a lessened ability to see conventional reality, as indicated by a positive Schizophrenia Index. This finding also suggests that these children have faulty or distorted reasoning that further impairs their functioning. The Hypervigilance Index (HVI) suggests that they invest significant energy in protecting the self against the perceived malevolence of the outside world. When combined with poor reality testing, hypervigilance becomes paranoia. They are interpersonally guarded, remain suspicious of others' motives, and zealously maintain personal space. Their relationships are likely to be distant and superficial. The HVI constellation is extremely rare in non-patient children and adolescents yet characterized the NPD children older than 7 years. Three of the four age groups also were positive on the Coping Deficit Index (CDI), a measure of general coping capacity, particularly in the interpersonal realm. A positive CDI is associated with interpersonal ineptness and a chaotic history of relationships. The Depression Index was also elevated in the NPD population, indicative of frequent experiences of dysphoric affect, low selfesteem, and psychological pain that was not consistently evident in the presenting symptoms of these patients.

These constellations are not only rare in the non-patient comparison group but also represent premature, crystallized, developmental formations that are stable over time and unlikely to disappear with continued maturation.

The impact on affect and cognitive processes is also profound as the results suggest. The elevated space responses are associated with an alienated, oppositional stance toward others that can lead to clashes with the environment. Anger and resentment are easily triggered, and the personal investment in being a nonconformist further distances these children from others. These children are very uncomfortable and avoidant around 
emotionally charged situations. They try to remove themselves from affective exchanges with others, as indicated by the low Affectivity Ratio scores. They strive to restrict and inhibit emotional expression in a manner that is not only atypical developmental for children and adolescents but also detrimental to learning from emotional exchanges with peers. In contrast with the non-patient group, the overall acknowledgment of feelings is suppressed in the Rorschach protocols as shown by the lower Weighted Sum C scores. The elevation in Morbid scores in the NPD group may provide a clue to the overall affective restriction. Morbid responses are associated with feelings of damage, pessimism, and inadequacy that may prove intolerable to NPD children who are valiantly battling to maintain their inflated but flawed sense of self.

\section{FURTHER VARIABLES}

The cognitive processes also appear to succumb to the need to protect the self. These children's Lambda scores are relatively high. Lambda represents a cognitive operation of reducing complex or ambiguous detail or information to a simplistic, "black and white" picture. This "just the facts, Ma'am," approach to life eliminates problematic nuances that may blur the situation. NPD children need to see problems in ways that suit their psychological needs. However, the simplification of facts, consequently, leaves them vulnerable to missing important (and possibly distressing) details. The increased X-percentage confirms that NPD children regularly distort information and are unable or unwilling to see things in conventional ways, was observed in the lower Popular scores. They engage in a problem solving style, and pervasively so, that is unexpected for children across the age range. This "Superintroversive" problem solving style involves delaying any reaction until alternatives are examined methodically, without integrating emotional cues.
The children turn to their own ideation as their best resource to solve problems or address demands. However, their susceptibility to simplifying and distorting information seriously compromises the efficiency of their problem-solving style.

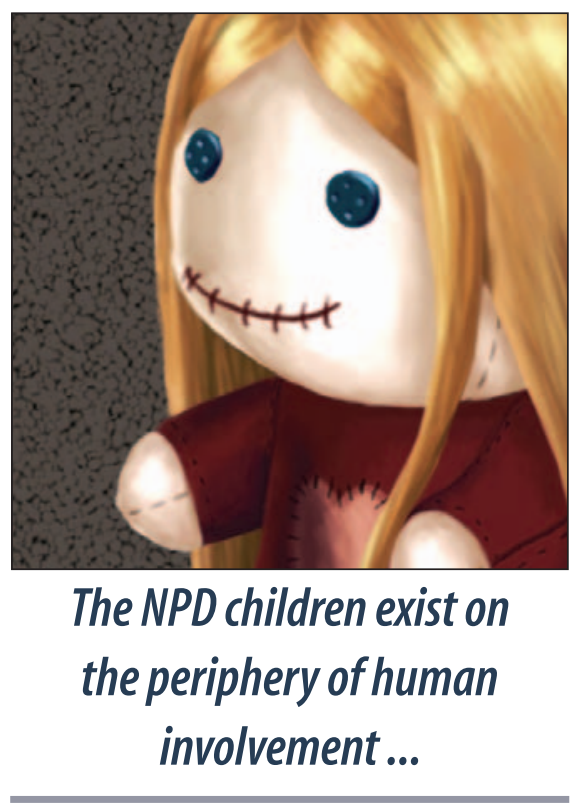

They are likely to generate unexpected and often inappropriate solutions to the demands that they experience.

The two Ideation variables confirm this problem. The NPD group engages in fantasy to an excess to avoid dealing with unpleasant situations, hoping that, eventually, they can escape any responsibility and someone else will "clean up the mess." This maladaptive "Snow White" syndrome, a term used to describe Mp > Ma scores, occurred two to four times more often in the NPD group than in the non-patient controls. The second variable, M-, also occurred more often in the NPD group. M- involves the use of human movement with distorted form and is empirically associated with circumscribed peculiar ideation about people that can become delusional.

Not surprisingly, the interpersonal realm is affected. The HVI score already suggests that the NPD group will keep their distance from others, who are always suspect. Further examination of the interpersonal variables reveals that, like most other patient populations, the absence of texture responses frequently found in this group, suggests a deficit in the ability to form attachments to others and a resignation to not having one's needs for nurturing met. The relative absence of aggressive and cooperative movement is also significant because these groups both share an interactive and mutual engagement, either positive or negative, that NPD children do not exhibit. The NPD children exist on the periphery of human involvement, observing but not truly connecting with others. The last variable, the overall number of full human responses, confirms the relative lack of interest and identification with other people. Others are perceived as extensions of the self or need-gratifiers or frustraters. People are objects to be used, envied, or devalued.

The findings in the self-perception variables are also consistent with the theoretical understanding of NPD. These patients exist with an inflated sense (as seen by their reflection responses) that exceed the non-patient's profile. Ironically, even the self-aggrandizement does not elevate their insidious feelings of inadequacy (Egocentricity ratio) that they try to keep from their own awareness. The presence of reflection responses also includes other operations such as denial and externalization of blame in an effort to preserve the need for a flawless self.

The unusual amount of Form Dimension (FD) responses in these children is an unexpected finding. The term refers to using contours to indicate depth. In adults, FD correlates with an ability to objectively look at oneself but in these children, the presence of FD may have a distinct meaning. It may indicate a precocious awareness of the self as others see one (or how one would wish to be seen by others) that is a precursor to the reflection response. This self-consciousness, ironically, emerges instead of the capacity for self-reflection or introspection, a person- 
ality trait that is rare in the child and adolescent NPD group. FD appears entwined with the HVI index as others are monitored to determine how the NPD child is being perceived. Stegge and Bushman ${ }^{5}$ found that the self esteem of narcissistic children is highly dependent on external evaluations: "Narcissists gain and lose worth according to how others view them ... In contrast, normal, healthy forms of self esteem are stable and relatively independent of the appraisals of others." As the sample has grown, the elevated FD has consistently appeared in the NPD children's Rorschach responses, without evidence of objective self-evaluation implied by adult studies. ${ }^{45}$ This need to monitor others' reactions to one's image is consistent with theory of NPD.

The structural features outlined in this study have a clinical counterpart in the content of these Rorschach protocols. The responses were classified according to theory and other research findings. The theory-based categories that were significantly present in these children's responses included grandiosity, exhibitionism, and the defenses of devaluation and idealization. Grandiose responses included percepts in which status and admiration were emphasized: "a preacher lifting his hands as he speaks to the people." Exhibitionistic responses included percepts in which the subject was trying to impress others: a girl in a bra and royal cape, shaking her pom-poms." Devaluation and idealization characterized responses in which the NPD patient criticized the subject of the response or the inkblots, themselves and elevated other responses as perfect or exemplary, respectively: "a stupid-looking moth, who did they get to make these dumb pictures, anyway?"; "a beautiful princess on a pure white stallion."

The theoretical constructs, according to the literature, that underlie NPD also include a dependency on others while maintaining an illusion of self-sufficiency; hypervigilance in anticipation of oth- ers' malevolence (aggression externalized onto others); and sadistic or aggressive pleasure in the defeat of others, without remorse or concern. These constructs were organized into the categories of food responses (Fd) which correlate with dependency, preparedness (content involving

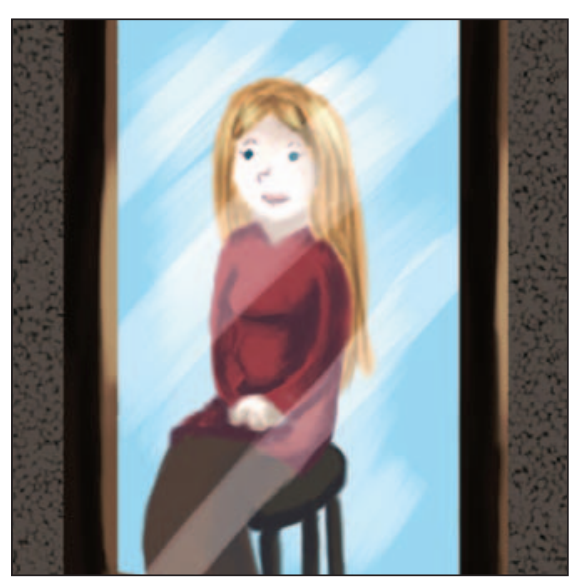

\section{The cruel, antisocial tendency associated with NPD is evident in these children's responses.}

radar, antennae, binoculars, weaponry), and aggressive or aggressive/morbid responses. As noted previously, cooperative or mutual relationships between subjects was unusual in the protocols. Interactions were often either dependent, destructive, or exploitative.

These NPD children and adolescents were often gleeful in their depiction of aggressive and morbid content. The cruel, antisocial tendency associated with NPD is evident in these children's responses.

Over half the protocols were characterized by depictions of "gross" or damaged content: The sense of repulsion and damage underlying the inflated sense of self in the content is consistent with theoretical predictions.

\section{SUMMARY}

The current study is a descriptive effort to determine the nature of NPD in children and to address if any variables are consistent with theory about narcissism and with the assumption that personality disorder exists in children. The findings indicate the narcissistic children and adolescents are presenting with pervasive personality pathology. They exhibit distortion of reality, constriction of affect, a hypervigilant wariness of others' motives, depressive features not apparent in the clinical presentation, inflated sense of self in the presence of a sense of inferiority and comparatively less interest in others, and less involvement in close relationships. They rely on themselves as their own best resources and engage little with others to solve problems. Their personality traits tend to stable, despite their maladaptive nature, and they are not likely to experience any need to engage in treatment to change the nature of their adjustment. Therapy is likely to trigger the paranoid and narcissistic traits as their behavior is examined and interpreted. These features are not only consistent with clinical descriptions of these patients outlined at the start of this chapter but also suggest the chronicity and intractability of the personality constellation that has been erected to compensate for the cracked image of the self.

\section{REFERENCES}

1. American Psychiatric Association. Diagnostic and Statistical Manual for Mental Disorders. 4th ed. Washington, D.C.: American Psychiatric Publishing; 1994.

2. Kernberg P, Weiner A, Bardenstein K. Personality Disorders in Children and Adolescents. New York, NY: Basic Books; 2000.

3. Harter S. The Construction of the Self: A Developmental Perspective. New York, NY: Guilford Press; 1991.

4. Thomaes S, Bushman BJ, Stegge H, Olthof T. Trumping shame by blasts of noise: Narcissism, self-esteem, shame, and aggression in young adolescents. Child Dev. 2008;79(6):1792-1801.

5. Stegge G, Bushman BJ. Narcissism, shame and aggression in early adolescence. PI Research Dulvendrecht. Amsterdam: Reprographie Vrije Universiteit; 2007.

6. Barry CT, Frick PJ, Killian AL. The relation of narcissism and self-esteem to conduct problems in children: a preliminary investigation. $J$ Clin Child Adolesc Psychol. 2007;32(1):139-152. 
7. Bleiberg E. Developmental pathogenesis of narcissistic disorders in children. Bull Menninger Clin. 1998;52(1):3-15.

8. Bleiberg E. Normal and pathological narcissism in adolescence. Am J Psychother. 1994;48(1):30-51.

9. Bleiberg E. Treating Personality Disorders in Children and Adolescents. A Relational Approach. New York, NY: Guilford Press; 2001

10. Kernberg PF. Narcissistic personality disorder in childhood. Psychiatr Clin North Am. 1989;12(3):671-694.

11. Kernberg O. Borderline Conditions and Pathological Narcissism. New York, NY: Jason Aronson; 1975.

12. Horton RS, Bleau G, Drwecki B. Parenting narcissus: what are the links between parenting and narcissism? J Pers. 2006;74(2):345-376.

13. Egan J, Kernberg PF. Pathological narcissism in childhood. Journal of the American Psychoanalytic Association. 1984;39-62.

14. Ferreira T. The all-powerful child: some aspects of narcissistic pathology. Revista Portuguesa de Psicanalise. 1990;8:61-70.

15. Rinsley DB. The severely disturbed adolescent: Indications for hospital and residential treatment. Bull Menninger Clin. 1990;54(1):3-12.

16. Cohen Y. Grandiosity in children with narcissistic and borderline personality disorders. A comparative analysis. Psychoanal Study Child. 1991;46:307-324.

17. Beren P. Narcissistic disorders. Psychoanal Study Child. 1992;47:265-278.

18. Bernstein JS. The grandiose character, primary type. Psychoanal Rev. 1995;82(2):293-311.

19. Imbesi L. On the etiology of narcissistic personality disorder in children and adolescents. Issues in Psychoanalytic Psychology. 2000;22(2):43-58.

20. Guile JM. Identifying personality disorders in pre-adolescents. Canadian Journal of Psychiatry. 1996;41(6):343-349.

21. Eppright TD, Kashani JH, Robinson BD, Reid JC. Comorbidity of conduct disorder and personality disorder in an incarcerated juvenile population. Am J Psychiatry. 1993;150(8):1233-1236.

22. Myers WC, Burket RC, Harris HE. Adolescent psychopathy in relation to delinquent behaviors, conduct disorders, and personality disorders. $J$ Forensic Sci. 1995;40(3):435-439.
23. Guile JM. Associated variables to narcissistic personality disorder in children and adolescents. Annales Medico-Psychologiques. 2002;160(8):550-558.

24. Guile JM. Initial reliability of the Diagnostic Interview for Narcissism adapted for pre-adolescents: Parent Version (P-DIN). Canadian Academy of Child and Adolescent Psychiatry Review. 2004;13(3):74-80.

25. Raskin R, Hall CS. The Narcissistic Personality Inventory: alternate from reliability and further evidence of its construct validity. J Pers Assess. 1981;45(2):159-162.

26. Aalsma M, Lapsley D, Flannery D. Personal fables, narcissism and adolescent adjustment. Psychology in the Schools. 2006;43(4):481-491.

27. Ang R, Yusof N. Reliability, validity and invariance of the Narcissistic Personality Questionnaire for Children-Revised (NPQC-R). Journal of Psychopathology and Behavioral Assessment. (Online publication). December 3, 2008.

28. Thomaes S, Stegge H, Bushman B, Olthof T, Denissen J. Development and validation of the Childhood Narcissism Scale. J Pers Assess. 2008;90(4):382-391.

29. Gunderson JG, Ronningstam E, Bodkin A. The diagnostic interview for narcissistic patients. Arch Gen Psychiatry. 1990;47(7):676-680.

30. Barry CT, Grafeman SJ, Adler KK, Pickard JD. The relations among narcissism, self-esteem, and delinquency in a sample of at risk adolescents. Journal of Adolescence. 2007;30(6):933-942.

31. Ang R, Yusof N. The relationship between aggression, narcissism, and self-esteem in Asian children and adolescents. Current Psychology. 2005;24 (2):113-122.

32. Barry T, Thompson A, Barry C, Lochman J, Adler K, Hill K. The importance of narcissism in predicting proactive and reactive aggression in moderately to highly aggressive children. Aggress Behav. 2007;33(3):185-197.

33. Crawford TN, Cohen P, Brook JS. Dramatic-erratic personality disorder symptoms: II. Developmental pathways from early adolescence to adulthood. J Pers Disord. 2001;15(4):336-350.

34. Abrams D. Pathological narcissism in an eight year old boy. An example of Bellak's TAT and CAT diagnostic system. Psychoanalytic Psychology. 1993;10(4):573-591.
35. Hilsenroth M, Fowler JC, Padawer J, Handler L. Narcissism in the Rorschach revisited; Some reflections on empirical data. Psychological Assessment. 1997;9:(2)113-121.

36. Exner J. A Rorschach Workbook for the Comprehensive System. Vol. 1. Basic Foundations. 3rd ed. New York: Wiley and Sons; 1990

37. Kwawer J. Primitive interpersonal modes, borderline phenomena and Rorschach content. In: Kwawer J, Lerner H, Lerner P, Sugarman A, eds. Borderline Phenomena and the Rorschach Test. New York, NY: International Universities Press, Inc.; 1980.

38. Lerner P, Lerner H. Rorschach assessment of primitive defenses in borderline personality structure. In: Kwawer J, Lerner $\mathrm{H}$, Lerner P, Sugarman A, eds. Borderline Phenomena and the Rorschach Test. New York: International Universities Press, Inc.; 1980.

39. Cooper S, Perry J, Arnow D. An empirical approach to the study of defense mechanisms. Reliability and preliminary validity of the Rorschach defense scales. J Pers Assessment. 1988;52(2):187-203.

40. Berg M. Borderline psychopathology as displayed on psychological tests. J Pers Assess. 1983;47:120-133.

41. Wagner EE, Hoover T. Behavioral implications of Rorschach's human movement response. Further validation based on exhibitionistic M's. Perceptual Motor Skills. 1972;35:27-30.

42. Bardenstein K. Rorschach features of narcissistic personality in children. In: Kernberg O, Hartmann HP, eds. Narzissmus. Stuttgart, Germany: Schattauer; 2006.

43. Hare JD. The Hare Psychopathy ChecklistRevised (PCC-R). Toronto, Canada: MultiHealth Systems; 1991.

44. Exner J. The Rorschach: A Comprehensive System. Vol. 1. Basic Foundations and Principles of Interpretation. 4th ed. Hoboken, NJ: Wiley and Sons; 2003

45. Bardenstein K. The unexpected persistence of form dimension in NPD children: It's not your father's FD. Presented at the Society for Personality Assessment meeting, Washington, DC; 2007. 